\title{
TERMINOLOGÍA Y FRASEOLOGÍA EN LOS DOCUMENTOS MÉDICO-LEGALES (2): EXTRACCIÓN, CLASIFICACIÓN, ANÁLISIS Y \\ TRADUCCIÓN DE UNIDADES DE NATURALEZA JURÍDICA Ana Belén Martinez López. \\ Universidad de Granada
}

\section{ABSTRACT}

This article intends to address the issue of translation posed by medicolegal documents of the English-Spanish combination. The study being conducted, in this case is based on the fieldwork based on a translation assignment of 30 forms and standard documents in relation to assisted reproduction. The final result focusing on the terminological units and phraseologisms most representative of the reference corpus discussed is a Spanish-English bilingual glossary of legal terms in relation to assisted reproduction.

KEYWORDS: legal translation, English-Spanish legal translation, legal terms in relation to assisted reproduction, phraseologisms.

\section{RESUMEN}

En este artículo pretendemos abordar la problemática de traducción que presentan los documentos médico-legales dentro de la combinación lingüística español-inglés. En este caso, el estudio realizado se basa en un trabajo de campo realizado a partir de un encargo profesional de traducción compuesto de 30 formularios y documentos-tipo relacionados con el ámbito de la reproducción asistida. El resultado final, centrado en las unidades terminológicas y fraseológicas más representativas del corpus de referencia analizado, es un glosario bilingüe de términos jurídicos (español-inglés) relacionados con la reproducción asistida.

PALABRAS CLAVE: traducción jurídica, traducción jurídica (español-inglés), términos jurídicos relacionados con la reproducción asistida, unidades fraseológicas. 
En este artículo vamos a proceder al análisis de las unidades fraseológicas y de las fórmulas hechas de naturaleza jurídica y jurídicoadministrativa de un tipo específico de documentos-tipo, los denominado documentos médico-legales. Para ello nos vamos a basar en un encargo de traducción profesional realizado por la autora de este trabajo durante los meses de marzo, abril y mayo de 2007 para la Unidad de Reproducción Asistida (URE) de un centro sanitario especializado de Málaga.

A este respecto, vamos a obviar lo expuesto en el artículo publicado en este mismo número de la revista (cf. "Terminología y fraseología en los documentos médico-legales (1): extracción, clasificación, análisis y traducción de unidades de naturaleza biosanitaria") para centrarnos exclusivamente en la extracción, clasificación y análisis de unidades de naturaleza jurídica y jurídicoadministrativa.

\section{EXTRACCIÓN Y CATALOGACIÓN DE UNIDADES FRASEOLÓGICAS (DEL ÁMBITO JURÍDICO) CONTENIDAS EN LOS DOCUMENTOS OBJETO DE TRADUCCIÓN}

La extracción de unidades fraseológicas (de temática jurídica) de los 30 documentos analizados dio el siguiente resultado (organizado por orden alfabético):

\section{RELACIÓN DE ENTRADAS}

A

A los fines citados anteriormente

A rellenar por el donante

Aspectos legales que se han de tener en cuenta

Autorizo al personal de la URE a

Autorización de sometimiento a pruebas y donación

Autorizaciones

Autorización para destrucción de muestras de semen/biopsia testicular (congeladas)

Autorización para traslado de muestras de semen

Autorización para traslado de embriones

Autorización-Consentimiento informado sobre descongelación de embriones

Autorizaciones-consentimientos informados 
C

Carácter anónimo y secreto de la donación

Comunidad Autónoma

Consentimiento informado para la anestesia

Consentimiento informado para conservación y almacenamiento de muestras de biopsia testicular

Consentimiento informado para inseminación artificial con semen de donante

Consentimiento informado para descongelación y transferencia embrionaria

Consentimiento informado para fecundación in vitro y transferencia embrionaria

Consentimiento informado para técnica quirúrgica

Consentimiento informado para inseminación artificial / fecundación in vitro / microinyección espermática (VIH, VHC, VHB)

Consentimiento informado para conservación y almacenamiento de muestras de semen

Consentimiento informado para programa de diagnóstico genético preimplantatorio

Consentimiento informado para tratamiento inductor de la ovulación

Consentimiento informado para inseminación artificial con semen de la pareja

Consentimiento informado para tratamiento inductor de la ovulación

Consentimientos informados

Contratos

Contrato sobre donación de embriones (mujer/pareja receptora)

Contrato de almacenamiento y custodia de embriones congelados (extraclínico)

Contrato de almacenamiento y mantenimiento de semen congelado

Contrato de reserva de semen de donante en la URE CENTRO GUTENBERG

Contrato de almacenamiento y mantenimiento de semen/BT congeladas (extraclínico)

Contrato-Consentimiento informado sobre donación de embriones (investigación)

Contrato-Consentimiento informado sobre donación de ovocitos (mujer receptora)

Contrato-Consentimiento informado sobre donación de ovocitos (donante)

Contrato-Consentimiento informado sobre donación de embriones (fines reproductivos)

Contrato-Consentimiento informado sobre donación de semen (donante)

Contratos-consentimientos informados

$\mathbf{E}$

En caso de incapacidad temporal del paciente

En nombre y representación de la Unidad de Reproducción Asistida Centro Gutenberg 
$\mathbf{F}$

Fecha $(\mathrm{d} / \mathrm{m} / \mathrm{a})$

Firma del doctor

Firma de la paciente

Firma de la pareja

Firma del representante legal

$\mathbf{H}$

He sido informado de las disposiciones siguientes de la ley sobre técnicas de reproducción asistida

I

Instituto Nacional de la Salud

Irrevocabilidad de la donación

$\mathbf{L}$

Ley 14/2006 sobre Técnicas de Reproducción Asistida

$\mathbf{M}$

Manifiesta que

Me comprometo a

Me hago responsable de las consecuencias que pudieran derivarse de mi decisión

Ministerio de Sanidad y Consumo

$\mathbf{N}$

Nombre y apellidos de la paciente

Nombre y apellidos de la pareja

Nombre y apellidos del representante legal

Número de hijos biológicos

Número del DNI o Pasaporte

$\mathbf{P}$

Parentesco

$\mathbf{R}$

Registro Nacional de Donantes de Gametos y Preembriones con fines de reproducción humana

Requisitos generales para ser donante de semen

Revocabilidad de la autorización 
Según el derecho que otorga la ley

Sociedad Española de Anestesiología y Reanimación

Sociedad Española de Alergología e Inmunología Clínica

Sometimiento a estudios previos

$\mathbf{U}$

Una vez leído y comprendido lo anterior quedo informado de

$\mathbf{Y}$

Yo, manifiesto de forma libre y consciente mi revocación del consentimiento informado aceptado con fecha

3. CLASIFICACIÓN DE LAS UNIDADES FRASEOLÓGICAS OBTENIDAS (DEL ÁMBITO JURÍDICO) POR CATEGORÍAS Y ANÁLISIS CUANTITATIVO Y CUALITATIVO DE RESULTADOS

Una vez analizado el material obtenido en el proceso de extracción, hemos establecido una serie de categorías que permiten dividir el material obtenido por temas más o menos independientes entre sí.

\subsection{LA DEFINICIÓN DE LAS CATEGORÍAS Y DE LAS UNIDADES FRASEOLÓGICAS} (DEL ÁMBITO JURÍDICO) QUE SE RECOGEN EN LOS DOCUMENTOS ANALIZADOS

Hemos establecido una división en cinco categorías que responden a los ámbitos siguientes:

CATEGORÍA 1. UNIDADES FRASEOLÓGICAS I (documentos-tipo). Unidades extraídas: 5.

En esta categoría se recogen las unidades fraseológicas que identifican los documentos-tipo que componen este encargo.

CATEGORÍA 2. UNIDADES FRASEOLÓGICAS II (documentos específicos). Unidades extraídas: 27.

En esta categoría se recogen las unidades fraseológicas que responden a los documentos específicos, de temática jurídica, que han sido objeto de traducción en este encargo.

CATEGORÍA 3. UNIDADES FRASEOLÓGICAS III (Identificación de las partes).

Unidades extraídas: 10. 
En esta categoría se recogen las unidades fraseológicas relacionadas con la "identificación de las partes", apartado éste que es común a todos los documentos específicos analizados.

\section{CATEGORÍA 4. UNIDADES FRASEOLÓGICAS IV (Fórmulas y} expresiones hechas). Unidades extraídas: 19.

En esta categoría se recogen las unidades fraseológicas relacionadas con el uso de "fórmulas" y "expresiones hechas", que es muy frecuente en la mayoría de los documentos específicos analizados.

CATEGORÍA 5. REFERENCIAS CULTURALES (Legislación e instituciones españolas). Unidades extraídas: 7.

En esta categoría incluimos las referencias culturales que aparecen contenidas en los documentos objeto de análisis. Al tratarse de un número reducido de entradas, las hemos incluido en un único apartado titulado "legislación e instituciones españolas".

3.2. El RESUlTADO DE LA EXTRACCIÓN DE UNIDADES FRASEOLÓGICAS (DEL ÁMBITO JURÍDICO) POR CATEGORÍAS

La extracción de unidades fraseológicas del ámbito jurídico (incluyendo en ellas las procedentes del análisis de los encabezamientos) divididas por categorías, dio el resultado siguiente:

\subsubsection{Categoría 1. Unidades fraseológicas I (documentos-tipo)}

Unidades extraídas: 5

\section{RELACIÓN DE ENTRADAS}

Autorizaciones

Autorizaciones-consentimientos informados

Consentimientos informados

Contratos

Contratos-consentimientos informados

\subsubsection{Categoría 2. Unidades fraseológicas II (documentos específicos)}

Unidades extraídas: 26 
RELACIÓN DE ENTRADAS

Autorización de sometimiento a pruebas y donación

Autorización para destrucción de muestras de semen/biopsia testicular (congeladas)

Autorización para traslado de muestras de semen

Autorización para traslado de embriones

Autorización-Consentimiento informado sobre descongelación de embriones

Consentimiento informado para la anestesia

Consentimiento informado para conservación y almacenamiento de muestras de biopsia testicular

Consentimiento informado para inseminación artificial con semen de donante

Consentimiento informado para descongelación y transferencia embrionaria

Consentimiento informado para fecundación in vitro y transferencia embrionaria

Consentimiento informado para técnica quirúrgica

Consentimiento informado para inseminación artificial / fecundación in vitro / microinyección espermática (VIH, VHC, VHB)

Consentimiento informado para conservación y almacenamiento de muestras de semen

Consentimiento informado para programa de diagnóstico genético preimplantatorio

Consentimiento informado para tratamiento inductor de la ovulación

Consentimiento informado para inseminación artificial con semen de la pareja

Contrato sobre donación de embriones (mujer/pareja receptora)

Contrato de almacenamiento y custodia de embriones congelados (extraclínico)

Contrato de almacenamiento y mantenimiento de semen congelado

Contrato de reserva de semen de donante en la URE CENTRO GUTENBERG

Contrato de almacenamiento y mantenimiento de semen/BT congeladas (extraclínico)

Contrato-Consentimiento informado sobre donación de embriones (investigación)

Contrato-Consentimiento informado sobre donación de ovocitos (mujer receptora)

Contrato-Consentimiento informado sobre donación de ovocitos (donante)

Contrato-Consentimiento informado sobre donación de embriones (fines reproductivos)

Contrato-Consentimiento informado sobre donación de semen (donante)

\subsubsection{Categoría 3. Unidades fraseológicas III (identificación de las partes)}

Unidades extraídas: 10 


\section{RELACIÓN DE ENTRADAS}

Nombre y apellidos de la paciente

Nombre y apellidos de la pareja

Nombre y apellidos del representante legal

Número de hijos biológicos

Firma del doctor

Firma de la paciente

Firma de la pareja

Firma del representante legal

Parentesco

Número del DNI o Pasaporte

\subsubsection{Categoría 4. Unidades fraseológicas IV (fórmulas y expresiones hechas)}

Unidades extraídas: 19

\section{RELACIÓN DE ENTRADAS}

A los fines citados anteriormente

A rellenar por el donante

Aspectos legales que se han de tener en cuenta

Autorizo al personal de la URE a

Carácter anónimo y secreto de la donación

En caso de incapacidad temporal del paciente

En nombre y representación de la Unidad de Reproducción Asistida Centro Gutenberg

Fecha $(\mathrm{d} / \mathrm{m} / \mathrm{a})$

He sido informado de las disposiciones siguientes de la ley sobre técnicas de reproducción asistida

Irrevocabilidad de la donación

Manifiesta que

Me comprometo a

Me hago responsable de las consecuencias que pudieran derivarse de mi decisión

Requisitos generales para ser donante de semen

Revocabilidad de la autorización

Según el derecho que otorga la ley

Sometimiento a estudios previos

Una vez leído y comprendido lo anterior quedo informado de

Yo, , manifiesto de forma libre y consciente mi revocación del consentimiento informado aceptado con fecha 
ENTREC ULTURAS Número 1. ISSN: 1989-5097. Fecha de public ación: 27-03-2009

3.2.5. Categoría 5. Referencias culturales (legislación e instituciones españolas)

Unidades extraídas: 7

RELACIÓN DE ENTRADAS

Comunidad Autónoma

Instituto Nacional de la Salud

Ley 14/2006 sobre Técnicas de Reproducción Asistida

Ministerio de Sanidad y Consumo

Registro Nacional de Donantes de Gametos y Preembriones con fines de reproducción humana

Sociedad Española de Anestesiología y Reanimación

Sociedad Española de Alergología e Inmunología Clínica

\section{ANÁLISIS CUANTITATIVO Y CUALITATIVO DE RESULTADOS POR CATEGORÍAS (ÁMBITO JURÍDICO)}

Un análisis cuantitativo de resultados, por importancia relativa de las categorías (número de entradas que incluye cada una de ellas), nos ofrece la siguiente tabla:

\begin{tabular}{|ll|c|}
\hline CATEGORÍAS & $\begin{array}{c}\text { Número de } \\
\text { unidades }\end{array}$ \\
\hline $\begin{array}{l}\text { CATEGORÍA 2. UNIDADES } \\
\text { (documentos específicos) }\end{array}$ & FRASEOLÓGICAS II & 26 \\
\hline $\begin{array}{l}\text { CATEGORÍA 4. UNIDADES } \\
\text { (Fórmulas y expresiones hechas) }\end{array}$ & FRASEOLÓGICAS IV & 19 \\
\hline $\begin{array}{l}\text { CATEGORÍA 3. UNIDADES } \\
\text { (Identificación de las partes) }\end{array}$ & FRASEOLÓGICAS III & 10 \\
\hline $\begin{array}{l}\text { CATEGORÍA 5. REFERENCIAS CULTURALES } \\
\text { (Legislación e instituciones españolas) }\end{array}$ & 7 \\
\hline $\begin{array}{l}\text { CATEGORÍA 1. UNIDADES FRASEOLÓGICAS I } \\
\text { (documentos-tipo) }\end{array}$ & 5 \\
\hline TOTAL DE UNIDADES EXTRAÍDAS & 67 \\
\hline
\end{tabular}

Como podemos apreciar en un análisis somero de los resultados obtenidos (desde un punto de vista cuantitativo), destacan las unidades fraseológicas relacionadas con la identificación de documentos específicos (27 entradas).

En segundo lugar, destacan las unidades fraseológicas que hemos incluido dentro del apartado de fórmulas y expresiones hechas (con 19 
ENTREC ULTURAS Número 1. ISSN: 1989-5097. Fecha de public ación: 27-03-2009

entradas), seguidas de las unidades fraseológicas relacionadas con la "identificación de las partes" en los documentos de autorización, contratos o consentimientos informados (con 10 entradas). Le siguen en importancia las referencias culturales (con 7 entradas) y cierra esta relación la categoría relativa a la identificación de documentos tipo (con 5 entradas).

\section{ANÁLISIS CUANTITATIVO Y CUALITATIVO (COMPARADO) DE LAS UNIDADES FRASEOLÓGICAS EXTRAÍDAS (ÁMBITOS BIOSANITARIO Y JURÍDICO)}

Para llevar a cabo un análisis cuantitativo comparado de resultados de la extracción de unidades fraseológicas de los ámbitos jurídico (en este artículo) y biosanitario (cf. "Terminología y fraseología en los documentos médicolegales (1): extracción, clasificación, análisis y traducción de unidades de naturaleza biosanitaria") en el corpus de 30 documentos objeto de estudio, hemos elaborado una tabla que se compone de 2 columnas. En la de la izquierda aparecen los valores relativos obtenidos por las 5 categorías del ámbito biosanitario, ordenadas por orden descendente de número de entradas. En la de la derecha, aparecen, por su parte, los valores relativos obtenidos por las 5 categorías del ámbito jurídico, ordenadas de la misma forma.

Por último, se especifica el número total de unidades extraído en cada uno de los ámbitos en la última casilla de cada una de las columnas.

\begin{tabular}{|c|c|}
\hline $\begin{array}{l}\text { CATEGORÍAS - } \\
\text { Ámbito biosanitario }\end{array}$ & $\begin{array}{l}\text { CATEGORIAS - } \\
\text { Ámbito jurídico }\end{array}$ \\
\hline $\begin{array}{l}\text { CATEGORÍA } 5 . \\
\text { Unidades fraseológicas relacionados } \\
\text { con enfermedades, patologías o } \\
\text { procesos analizados y valorados en } \\
\text { diagnósticos genéticos previos u otros } \\
\text { protocolos de actuación } \\
\text { Unidades extraídas: } 60\end{array}$ & $\begin{array}{l}\text { CATEGORÍA } 2 . \\
\text { Unidades fraseológicas II documentos } \\
\text { específicos) } \\
\text { Unidades extraídas: } 26\end{array}$ \\
\hline $\begin{array}{l}\text { CATEGORÍA } 2 . \\
\text { Unidades fraseológicas relacionadas } \\
\text { con tratamientos, dispositivos y } \\
\text { técnicas de reproducción asistida } \\
\text { (quirúrgicas o no) } \\
\text { Unidades extraídas: } 41\end{array}$ & $\begin{array}{l}\text { CATEGORÍA } 4 . \\
\text { Unidades fraseológicas IV (Fórmulas } \\
\text { y expresiones hechas) } \\
\text { Unidades extraídas: } 19\end{array}$ \\
\hline $\begin{array}{l}\text { CATEGORÍA } 4 . \\
\text { Unidades fraseológicas relacionadas } \\
\text { con procesos específicos de los }\end{array}$ & $\begin{array}{l}\text { CATEGORÍA } 3 . \\
\text { Unidades fraseológicas } \\
\text { (Identificación de las partes) }\end{array}$ \\
\hline
\end{tabular}




\begin{tabular}{|c|c|}
\hline $\begin{array}{l}\text { programas de reproducción asistida } \\
\text { (reserva, donación, traslado, } \\
\text { congelación y descongelación de } \\
\text { embriones o semen) } \\
\text { Unidades extraídas: } 23\end{array}$ & Unidades extraídas: 10 \\
\hline $\begin{array}{l}\text { CATEGORÍA } 1 . \\
\text { Unidades fraseológicas relacionados } \\
\text { con la reproducción asistida (que } \\
\text { informan sobre las condiciones que se } \\
\text { han de dar para iniciar un programa } \\
\text { de reproducción asistida, sobre el } \\
\text { estado de la mujer y/o sobre las } \\
\text { consecuencias que pueden derivarse } \\
\text { de la aplicación de un programa de } \\
\text { este tipo) } \\
\text { Unidades extraídas: } 20\end{array}$ & $\begin{array}{l}\text { CATEGORÍA } 5 . \\
\text { Referencias culturales (Legislación e } \\
\text { instituciones españolas) } \\
\text { Unidades extraídas: } 7\end{array}$ \\
\hline $\begin{array}{l}\text { CATEGORÍA } 3 \text {. } \\
\text { Unidades fraseológicas relacionadas } \\
\text { con los tipos de anestesia y su } \\
\text { administración } \\
\text { Unidades extraídas: } 7\end{array}$ & $\begin{array}{l}\text { CATEGORÍA } 1 . \\
\text { Unidades fraseológicas I } \\
\text { (documentos-tipo) } \\
\text { Unidades extraídas: } 5\end{array}$ \\
\hline $\begin{array}{l}\text { TOTAL } \quad \text { DE } \\
\text { EXTRAÍDAS: } 151\end{array}$ & $\begin{array}{l}\text { TOTAL } \quad \text { DE } \\
\text { EXTRAÍDAS: } 68\end{array}$ \\
\hline
\end{tabular}

De un acercamiento somero a los resultados contenidos en la tabla anterior se deduce, desde una perspectiva cuantitativa, lo siguiente:

1) El número de entradas obtenido en el ámbito biosanitario es ostensiblemente superior al obtenido en el ámbito jurídico (151 entradas en el ámbito biosanitario frente a 67 en el jurídico).

2) En el ámbito biosanitario la categoría mejor representada no procede principalmente del análisis de los encabezamientos sino de unidades fraseológicas relacionadas con enfermedades, patologías o procesos analizados y valorados en diagnósticos genéticos previos u otros protocolos de actuación (con 60 entradas). Sin embargo, en el ámbito jurídico la categoría más amplia es la relativa a la identificación de documentos específicos (con 27 entradas), por lo que estas unidades fraseológicas se han obtenido principalmente del análisis de los encabezamientos de los 30 documentos del encargo. 
3) Otro aspecto cuantificable que se deriva del estudio comparado de ambas tablas de resultados nos permite afirmar que hay una mayor diversidad de unidades fraseológicas representativas del ámbito biosanitario, mientras que se da una mayor homogeneidad en las unidades jurídicas encontradas.

5. GLOSARIO DE UNIDADES FRASEOLÓGICAS (ESPAÑOLINGLÉS), DE NATURALEZA JURÍDICA, RELACIONADAS CON LA REPRODUCCIÓN ASISTIDA

Presentamos a continuación un glosario bilingüe que es el resultado de la comparación de unidades fraseológicas (relativas al ámbito jurídico y jurídico-administrativo, contratos y consentimientos informados) en los documentos del corpus analizado (textos originales y traducidos).

\begin{tabular}{|c|c|}
\hline ESPAÑOL & INGLÉS \\
\hline $\begin{array}{l}\text { 1. UNIDADES } \\
\text { FRASEOLÓGICAS I } \\
\text { (documentos-tipo) }\end{array}$ & $\begin{array}{l}\text { 1. UNIDADES } \\
\text { FRASEOLÓGICAS I } \\
\text { (documentos-tipo) }\end{array}$ \\
\hline $\begin{array}{l}\text { 1. Autorizaciones } \\
\text { 2. Autorizaciones-consentimientos } \\
\text { informados } \\
\text { 3. Consentimientos informados } \\
\text { 4. Contratos } \\
\text { 5. Contratos-consentimientos } \\
\text { informados }\end{array}$ & $\begin{array}{l}\text { 1. Informed consents } \\
\text { 2. Informed consents } \\
\text { 3. Informed consents } \\
\text { 4. Agreements } \\
\text { 5. Agreements-informed consents }\end{array}$ \\
\hline $\begin{array}{l}\text { 2. UNIDADES } \\
\text { FRASEOLÓGICAS II } \\
\text { (documentos específicos) }\end{array}$ & $\begin{array}{l}\text { 2. UNIDADES } \\
\text { FRASEOLÓGICAS II } \\
\text { (documentos específicos) }\end{array}$ \\
\hline $\begin{array}{l}\text { 6. Autorización para destrucción de } \\
\text { muestras de semen/biopsia testicular } \\
\text { (congeladas) } \\
\text { 7. Autorización para traslado de } \\
\text { muestras de semen } \\
\text { 8. Autorización para traslado de } \\
\text { embriones } \\
\text { 9. Autorización-Consentimiento }\end{array}$ & $\begin{array}{l}\text { 6. Informed consent for the } \\
\text { destruction of semen / testicular } \\
\text { biopsy samples (frozen) } \\
\text { 7. Informed consent to transfer semen } \\
\text { samples } \\
\text { 8. Informed consent to transfer } \\
\text { embryos } \\
\text { 9. Informed consent for defrosting }\end{array}$ \\
\hline
\end{tabular}




informado sobre descongelación de
embriones
10. Consentimiento informado para la
anestesia
11. Consentimiento informado para
conservación y almacenamiento de
muestras de biopsia testicular
12. Consentimiento informado para
inseminación artificial con semen de
donante
13. Consentimiento informado para
descongelación y transferencia
embrionaria

14. Consentimiento informado para fecundación in vitro y transferencia embrionaria

15. Consentimiento informado para técnica quirúrgica

16. Consentimiento informado para inseminación artificial / Fecundación in Vitro / microinyección espermática (VIH, VHC, VHB)

17. Consentimiento informado para conservación y almacenamiento de muestras de semen

18. Consentimiento informado para programa de diagnóstico genético preimplantatorio

19. Consentimiento informado para tratamiento inductor de la ovulación 20. Consentimiento informado para inseminación artificial con semen de la pareja

21. Contrato sobre donación de embriones (mujer/pareja receptora) 22. Contrato de almacenamiento y custodia de embriones congelados (extraclínico)

23. Contrato de almacenamiento y mantenimiento de semen congelado 24. Contrato de reserva de semen de donante en la URE Centro Gutenberg embryos

10. Informed consent for anaesthesia

11. Informed consent for the storage and preservation of testicular biopsy samples

12. Informed consent for artificial insemination with semen from a donor

13. Informed consent for embryo defrosting and transfer

14. Informed consent for in vitro fecundation and embryo transfer

15. Informed consent for surgical procedure

16. Informed consent for artificial insemination / in vitro fecundation / intracytoplasmatic sperm injection (HIV, HVC, HBV)

17. Informed consent for the storage and preservation of semen samples

18. Informed consent for the preimplantation genetic diagnosis program

19. Informed consent for treatment to induce ovulation

20. Informed consent for artificial insemination with semen from the partner

21. Agreement for embryo donation (recipient female / recipient couple) 22. Agreement for the storage and custody of frozen embryos (from other Medical Centres)

23. Agreement for the storage and preservation of frozen semen samples 24. Agreement to reserve semen from a donor at the Centro Gutenberg 
25. Contrato de almacenamiento y mantenimiento de semen/BT congeladas (extraclínico)

26. Contrato-Consentimiento informado sobre donación de embriones (investigación)

27. Contrato-Consentimiento informado sobre donación de ovocitos (mujer receptora)

28. Contrato-Consentimiento informado sobre donación de ovocitos (donante)

29. Contrato-Consentimiento informado sobre donación de embriones (fines reproductivos)

30. Contrato-Consentimiento informado sobre donación de semen (donante)

\section{UNIDADES}

\section{FRASEOLÓGICAS III}

(Identificación de las partes)

31. Nombre y apellidos de la paciente 32. Nombre y apellidos de la pareja

33. Nombre y apellidos del representante legal

34. Número de hijos biológicos

35. Firma del doctor

36. Firma de la paciente

37. Firma de la pareja

38. Firma del representante legal

39. Parentesco

40. Número del DNI o Pasaporte

\section{UNIDADES}

\section{FRASEOLÓGICAS IV}

(Fórmulas y expresiones hechas)

41. A los fines citados anteriormente

\section{Reproduction Unit}

25. Agreement to store and preserve frozen semen samples (from other Medical Centres)

26. Agreement-informed consent for embryo donation (research aims)

27. Agreement-informed consent for oocyte donation (female recipient)

28. Agreement-informed consent for oocyte donation (donor)

29. Agreement-informed consent for embryo donation (reproductive aims)

30. Agreement-informed consent for semen donation (donor)

\section{UNIDADES}

\section{FRASEOLÓGICAS III}

(Identificación de las partes)

31. Patient's full name

32. Partner's full name

33. Full name of the legal representative

34. Number of biological offspring

35. Doctor's signature

36. Patient's signature

37. Partner's signature

38. Signature of the legal representative

39. Relationship

40. Passport number

4. UNIDADES

FRASEOLÓGICAS IV

(Fórmulas y expresiones hechas)

41. In further regard of the aims 
42. A rellenar por el donante

43. Aspectos legales que se han de tener en cuenta

44. Autorizo al personal de la URE a

45.Autorización de sometimiento a pruebas y donación

46. Carácter anónimo y secreto de la donación

47. En caso de incapacidad temporal del paciente

48. En nombre y representación de la Unidad de Reproducción Asistida Centro Gutenberg

49. Fecha $(\mathrm{d} / \mathrm{m} / \mathrm{a})$

50. He sido informado de las disposiciones siguientes de la ley sobre técnicas de reproducción asistida

51. Irrevocabilidad de la donación

52. Manifiesta que

53. Me comprometo a

54. Me hago responsable de las consecuencias que pudieran derivarse de mi decisión

55. Requisitos generales para ser donante de semen

56. Revocabilidad de la autorización 57. Según el derecho que otorga la ley 58. Sometimiento a estudios previos 59. Una vez leído y comprendido lo anterior quedo informado de

60. Yo, manifiesto de forma libre y consciente mi revocación del consentimiento informado aceptado con fecha

\section{REFERENCIAS} CULTURALES mentioned above

42. To be filled in by the donor

43. Legal issues to be considered

44. I authorise the staff of the Reproduction Unit to

45. Authorisation to undergo tests and donation:

46. Anonymity and privacy of the donation

47. In case of patient's temporal inability

48. In the name and representation of the Centro Gutenberg Reproduction Unit

49. Date $(\mathrm{d} / \mathrm{m} / \mathrm{y})$

50. I have been informed of the following dispositions of the Ley sobre Técnicas de Reproducción Asistida [The Spanish Act on Techniques of Assisted Reproduction]

51. Irrevocability of the donation

52. Declares that

53. I oblige myself to

54. I am inherently responsible for the consequences deriving from my decision

55. General requirements for being a semen donor

56. Revocability of the authorisation 57. In view of the right granted by law 58. Subject to previous testing 59. After having read and understood the information above, I have been informed of

60. I, freely and consciously declare my revocation of the informed consent accepted on (date)

\section{REFERENCIAS CULTURALES}


ENTREC ULTURAS Número 1. ISSN: 1989-5097. Fecha de publicación: 27-03-2009

\section{(Legislación e instituciones españolas)}

\section{Comunidad Autónoma \\ 62. Instituto Nacional de la Salud}

63. The Spanish Act on Techniques of Assisted Reproduction

64. Ministerio de Sanidad y Consumo

65. Registro Nacional de Donantes de Gametos y Preembriones con fines de reproducción humana

66. Sociedad Española de

Anestesiología y Reanimación

67. Sociedad Española de Alergología e Inmunología Clínica
(Legislación e instituciones españolas)

\section{Autonomous Community}

62. Instituto Nacional de la Salud [Spanish National Health Institute] 63. Ley 14/2006 sobre Técnicas de Reproducción Asistida [The Spanish Act on Techniques of Assisted Reproduction]

64. Ministerio de Sanidad y Consumo [Spanish Health Department] 65. Registro Nacional de Donantes de Gametos y Preembriones con fines de reproducción bumana [Spanish National Registry of Gamete Donors and Preembryos with aims of human reproduction] 66. Sociedad Española de Anestesiología y Reanimación [Spanish Society for Anaesthesiology and Resuscitation] 67. Sociedad Española de Alergología e Inmunología Clinica [Spanish Society for Allergy and Clinical Immunology] 